\title{
International agricultural trade forecasting using machine learning
}

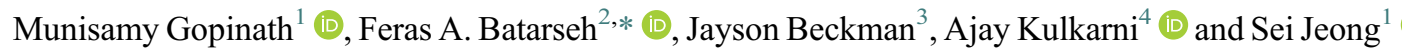 \\ ${ }^{1}$ Department of Agricultural and Applied Economics, University of Georgia, Athens, Georgia, USA \\ ${ }^{2}$ Bradley Department of Electrical and Computer Engineering, Virginia Polytechnic Institute and State University (Virginia Tech), \\ Arlington, Virginia, USA \\ ${ }^{3}$ Economic Research Service, U.S. Department of Agriculture, Washington, DC, USA \\ ${ }^{4}$ College of Science, George Mason University, Fairfax, Virginia, USA \\ *Corresponding author. E-mail: batarseh@vt.edu
}

Received: 31 August 2020; Revised: 17 November 2020; Accepted: 24 November 2020

Key words: agriculture; boosting algorithms; forecasting; machine learning; trade flows

JEL Classification: F14; C45; Q17

\begin{abstract}
Focusing on seven major agricultural commodities with a long history of trade, this study employs data-driven analytics to decipher patterns of trade, namely using supervised machine learning (ML), as well as neural networks. The supervised ML and neural network techniques are trained on data until 2010 and 2014, respectively. Results show the high relevance of ML models to forecasting trade patterns in near- and long-term relative to traditional approaches, which are often subjective assessments or time-series projections. While supervised ML techniques quantified key economic factors underlying agricultural trade flows, neural network approaches provide better fits over the long term.
\end{abstract}

\section{Policy Significance Statement}

Trade policy changes have become more frequent in the last few years, unlike their pattern prior to 2016. Predicting trade flows in this highly uncertain policy environment requires high-frequency and on-time data, as well as the appropriate techniques; such as machine learning, to provide critical and timely information to stakeholders. In this article, supervised models and neural networks are applied to forecasting agricultural trade patterns, and to identifying underlying contributors including policies. The impact of tariffs and multiple other economic variables on international trade is evaluated and presented.

\section{Introduction and Motivation}

Efficient and nimble agriculture and food industries are vital to human survival. In the past three years, global agriculture has been buffeted by many shocks - natural disasters, trade wars, and pandemics. Such unprecedented uncertainties have affected the range of decisions starting at the farm and culminating at the consuming household or ports (trade). Assessing such unconventional trends requires ample amounts of data. Over the past decade, increased availability of big data and breakthroughs in computer hardware have challenged conventional statistical and econometric techniques in modeling complex trends or 
economic relationships (Varian, 2014). The challenges include dealing with the sheer volume of data (evolving from spreadsheets to SQL databases, and recently, toward NoSQL and Hadoop clusters), the lengthy list of variables available to explain such relationships (and associated collinearity issues), and the need to move beyond simple linear models. Machine learning (ML) has been offered as an alternative to address many of these challenges (Bajari et al., 2015; Batarseh and Yang, 2017; Mullainathan and Spiess, 2017; Athey and Imbens, 2019). Several authors including Chief Economists of Google and Amazon have strongly advocated the use of big data and ML to uncover increasingly complex relationships even in an analysis as simple as fitting a supply or demand function. The economics community is catching on, but the speed of ML advances, that is, new techniques emerge frequently, can make an academic study stale by the time peer reviews are completed. Nonetheless, the academic community facing seismic shocks from advances in data and ML has been called on to revisit time-tested theories and relationships (Mullainathan and Spiess, 2017). This study takes on this challenge in the context of international trade and offers an ML application using agricultural trade data spanning several decades.

Many international institutions and government agencies project economic variables including trade flows to inform decisions in national and multilateral contexts (World Economic Outlook-International Monetary Fund, 2019; Trade in Goods and Services Forecast, Organization for Economic Cooperation and Development, 2019; U.S. Department of Agriculture, 2019; World Trade Organization, 2019). Since these projections are based on a combination of model-based analyses and expert judgment, several sources have pointed to their limitations, for example, forecast accuracy under $35 \%$ (U.S. Department of Agriculture, 2019) and quantifying the contribution of underlying economic factors (Chapter 4, World Economic Outlook-International Monetary Fund, 2019). With recent trade disruptions such as Brexit, USA-China/Japan-South Korea tariffs, the need for alternative approaches to understanding and forecasting (modeling) trade flows is greater now than ever before. During such disruptions, decisions to plant, maintain crop progress, harvest and market in the near term and to invest in farm assets in the medium term have all been impacted by serious supply-side disruptions (e.g., flooding or drought), significant uncertainty in demand (e.g., soybean purchases by China) and sudden collapse of both supply of inputs and demand for output (e.g., Covid-19). These events, especially in the context of agricultural trade, have created a level of uncertainty and complexity unknown over the past several decades. Compounding the situation is the static nature of most trade models, which often conduct comparative static analysis of trade outcomes from deterministic trade policy changes. Little guidance exists on theoretical modeling of trade policy uncertainty and its implications for producer and consumer preferences or behavior.

In this study, ML techniques are applied to the gravity model of bilateral (aggregate or industry) trade flows. The gravity model is often referred to as the workhorse in international trade due to its popularity and success in quantifying the effects of various determinants of international trade. Originally due to Anderson (1979) and applied to data in Anderson and van Wincoop (2003), the gravity model provides the causal association needed to implement ML algorithms in the predictive domain (Santos Silva and Tenreyro, 2006; Yotov et al., 2016; Athey and Imbens, 2019). In doing so, this study offers an alternative to time-series projections and expert judgment analyses by relying on neural networks and boosting approaches that allow for alternative and robust specifications of complex economic relationships (Baxter and Hersh, 2017; Storm et al., 2019). ML models can also provide accurate predictions, a priority of many economists in recent months given the trade disruptions among the major economies of the world.

\section{Machine Learning Methods}

Machine learning, a set of algorithms for advanced statistical analysis and intelligent problem solving, offers a novel approach (driven by big data sets) to identify patterns and model relationships, that is, quantify $Y$ 's response with or without a set $X$ of possible predictors, either supervised or unsupervised, respectively (James et al., 2013). Supervised learning (such as regression and classification) relates the response of $Y$ to $X$ for either better understanding of their relationship or predicting the response of $Y$ to a potential/future set of $X$. In contrast, unsupervised learning (such as clustering) usually does not have a 
pre-defined response variable $(Y)$ and aims to understand the relationships between/among $X$ or observations. In both settings, often relationships are derived from one or more "training" data sample and applied to a "test" data sample to compute prediction accuracy. The sheer volume of data available in recent times allows such a partition of data for cross-validation, that is, training and testing. However, a major trade-off arises between prediction accuracy and model interpretability between conventional (econometric) analysis and ML. As applicable techniques become non-linear or multi-layered with the repeated feedbacks between training and test data, ML techniques often leave interpretability and inference behind to focus more on data patterns and predictions accuracy. This study applies a variety of ML techniques to the trade setting noted earlier and also presents the inner working and challenges of ML in these settings.

A comprehensive review of all ML techniques available at this time is beyond the scope of this study. Excellent sources for that information include James et al. (2013), Batarseh and Yang (2017), and Athey and Imbens (2019). In the following, the generic optimization problem as in Athey and Imbens (2019) is presented along with an outline of techniques employed in this study. Consider a simple example where the outcome $Y$ depends on a set of features $X$. Assume:

$$
Y_{i} \mid X_{i} \sim N\left(\alpha+\beta^{T} X_{i}, \sigma^{2}\right),
$$

where $\theta=(\alpha, \beta)$ are parameters of interest, $Y_{i}$ has a conditional normal distribution with variance $\sigma^{2}$. Conventional econometric estimation, that is, least squares, would suggest:

$$
(\widehat{\alpha}, \widehat{\beta})=\arg \min _{\alpha, \beta} \sum_{i=1}^{N}\left(Y_{i}-\alpha-\beta^{T} X_{i}\right)^{2} .
$$

In the ML setting, the goal is usually to make a prediction for the outcome from a new set of values for $X$ that is, predicting $Y_{N+1}$ from $X_{N+1}$. Let that prediction, regardless of the actual specification of the relationship between $Y$ and $X$ be $\widehat{Y}_{N+1}$. Then, the squared loss associated with this prediction would be:

$$
\left(Y_{N+1}-\widehat{Y}_{N+1}\right)^{2}
$$

While "least squares" is an approach to minimize the loss function, other estimators exist that dominate least squares (Athey and Imbens, 2019). However, ML-based estimators for Equation (3) have a tendency to over or under-fit, which can be corrected by regularization, sampling, or tuning parameters through outof-sample cross-validation. ${ }^{1}$ The following provides a brief overview of ML techniques considered in this study:

- Ridge regression and elastic nets are some basic extensions to the least squares minimization problem in Equation (2) to impose a penalty for increasing the dimensionality of $X$ (i.e., regularization). A major concern with these approaches is the subjective choice on the penalty, often referred to as $\lambda$, but big data allow for its potential out-of-sample cross validation.

- Decision trees and their extensions have become extremely popular in recent years. They are referred to as trees since data are stratified or segmented into branches (splits) and leaves (nodes). The stratification is based on the number of predictors and cut-off values for predictors. For instance, if $X$ contains two column vectors, then stratification will be based on both constituents, sequentially or in random, for all possible cut-off values for each of these two predictors, for example, $X_{1}<c_{1}$. To make a prediction for new values of $X$, trees typically use the mode or median

\footnotetext{
${ }^{1}$ For qualitative data, a similar contrast exists between conventional logistic regression and approaches such as discriminant analysis and K-Nearest Neighbors. This study does not discuss qualitative data techniques in the following since the application employs quantitative data.
} 
of the outcome $Y$ in the region to which the new $X$ belongs. To illustrate, as in Athey and Imbens (2019), the total-sample sum of squared errors for outcome $Y$ is given by:

$$
Q=\sum_{i=1}^{N}\left(Y_{i}-\bar{Y}\right)^{2} \quad \bar{Y}=\sum_{i=1}^{N} Y_{i} .
$$

After a split based on one of the predictors $\left(X_{k}\right)$ using the threshold $X_{k}<c$, the sum of total-sample squared errors is:

$$
Q(k, c)=\sum_{i=X_{i k} \leq c}\left(Y_{i}-\bar{Y}_{k, c, l}\right)^{2}+\sum_{i=X_{i k}>c}\left(Y_{i}-\bar{Y}_{k, c, r}\right)^{2}
$$

where $l$ and $r$ denote left and right of $X_{k}$ using the cut-off $c$ and

$$
\bar{Y}_{k, c, l}=\frac{\sum_{i=X_{i k} \leq c} Y_{i}}{\sum_{i=X_{i k} \leq c} 1}, \bar{Y}_{1, c, r}=\frac{\sum_{i=X_{i k}>c} Y_{i}}{\sum_{i=X_{i k}>c} 1} .
$$

The objective of decision-tree based learning is to minimize $Q(k, c)$ for every $k$ and every $c \in(-\infty,+\infty)$, and the process is repeated for subsamples or leaves. As noted earlier, there is a tendency in this approach to over-fit, which can be corrected by using boosting, adding a penalty for the number of leaves, or by pruning the tree. A single tree is often the preferred outcome from this approach for its interpretability. However, prediction accuracy has been significantly improved (at the expense of interpretation) by procedures such as bagging, random forests, and boosting:

- Bagging involves repeated sampling of the (single) training data to fit a tree each. Then, averaging across trees chosen independently (like in bootstrapping) improves its prediction by lowering the variance.

- The random forests approach is similar to bagging in the sense that bootstrapped training samples are used to generate decision trees to average out. However, at each split of the decision tree, the choice on predictors (or a subset) is random.

- Boosting is also similar to bagging, but the decision tree for each training sample is not independent of previous trees, instead, they are chosen sequentially. Each tree is grown using information from previously grown trees and thus, boosting does not involve bootstrap sampling. Unlike bagging and random forests which are applicable to decision trees only, boosting can be employed for any base learner.

- Extra trees regression: this method deploys several trees for the same problem, and generates a mean of all the trees that reflects inclusion of all observations, and maximizes quality of the predictive outputs. That is, this method implements a meta-estimator that fits and averages a number of randomized trees to control over-fitting.

In the ML literature, the boosting algorithms are popular as they convert a weak base learner (often a single decision tree) into a strong learner (by re-training weak sub-samples as well as tuning of hyper parameters to provide optimized predictions).

- Neural networks and other deep learning methods are often employed in situations with large volumes of unexplored data to predict an outcome or analyze a pattern (especially used in image and audio recognition, as well as computer vision). These techniques emulate the human brain's neural system by recursively building multiple layers of nodes. These deep layers (thus referred to as deep learning) include predictors in linear form, but then translate them into latent variables and use nonlinear functional forms to relate to the outcome. To illustrate a single-layer network learning, consider the latent variables $Z$ defined as follows:

$$
Z_{i k}^{(1)}=\sum_{j=1}^{k} \beta_{k j}^{(1)} X_{i j}, \quad k=1, \ldots, K_{1} .
$$


Then, a non-linear function $g($.$) relates the outcome Y$ to $Z$ :

$$
Y_{i}=\sum_{k=1}^{K_{1}} \beta_{k}^{(2)} g\left(Z_{i k}^{(1)}\right)+\varepsilon .
$$

The objective of the estimation again is minimizing squared errors, but the layering allows for millions of functional possibilities and parameters. Note that such unsupervised ML techniques often do not have both outcomes $Y$ to $X$. In the context of Equations (6a) and (6b), the $X$ can be thought of some transformation of $Y$, for example, lagged values.

In this study, ML methods are considered for the standard specification of the gravity model:

$$
Y_{i j t}=g\left(X_{i t}, X_{j t}, i, j, t\right),
$$

where $Y_{i j t}$ is bilateral trade between country $i$ and country $j$ at time $t$, (response variable) and $X_{i t(j t)}$ is the set of possible predictors from both countries and the set $\{i, j, t\}$ refer to a variety of controls on all three dimensions (Anderson and van Wincoop, 2003; Yotov et al., 2016). The major ML techniques applied include random forests, extra tree regression, boosting, and neural networks. While it is tempting to compare ML models with econometric approaches, for example, the popular Poisson Pseudo-Maximum Likelihood (PPML) method commonly used to estimate trade flows, a word of caution is in order. ML techniques often encompass four paradigms - descriptive, diagnostic, predictive, and prescriptive — and the focus of this study is in the predictive domain. Nonetheless, we do present results from applying PPML estimates of the gravity model in Appendix I.

\section{The Agricultural Trade Setting and Data}

References to agricultural trade abundantly appear in literature dating back to 1000 BCE. One of the earliest pathways connecting Mediterranean to Arabia, Indian sub-continent, and Far East was the Incense Route. As the name suggests, incense made of aromatic plants and oils was a major traded commodity, but spices, silk, and precious stones were also major transactions along this route. Then came Spice and Silk Routes and numerous other inter- and intra-continental routes facilitating trade in agricultural products and other goods. The industrial revolution of 18th century favored agricultural industries, but that in late 19 th and early 20th century expanded rapidly into transport and energy sectors. Fast forward to the later parts of the 20th century, agriculture still accounted for at least a quarter of exports (or imports) of major trading nations. For instance, the 1960 edition of the State of Food and Agriculture from United Nations' Food and Agriculture Organization noted the significant share of agriculture in merchandise exports from the new world (North America and Oceania) to the rest of the world.

Anderson (2016) reviewing the evolution of food trade patterns over the past six decades, notes that agriculture's share of global merchandise trade was about $27 \%$ in 1960 . While that share has fallen to $11 \%$ in 2014 (due in part to lower prices of agricultural goods relative to industrial goods) the volume of agricultural exports has continued the strong upward trend of the early 20th century. Agricultural products are also unique in many aspects, for example, high volatility of prices, high level of trade protection, and immobility of countries' endowments such as land and water. More importantly, Anderson (2016) notes the concentration in both country and commodity shares of global exports of farm products. In particular, less than 10 items made up half of that trade in agricultural products and two-thirds of the world's exports of farm products are accounted by just a dozen trading economies. Despite that concentration, the set of commodities that stand out for having most countries involved in world trade are shown in Table 1. The seven commodities - wheat, corn, rice, sugar, beef, milk powder, and soybean - have not only been traded for long but also have the most countries engaged on the export or import side. Hence, this study chose to apply ML techniques to understand the patterns of agricultural trade where the longest time series and most country pairings exist, that is, the seven commodities in Table 1. 
Table 1. Number of exporting and importing countries of major agricultural products, 1970-2009.

\begin{tabular}{|c|c|c|c|c|c|c|c|c|}
\hline & \multicolumn{2}{|c|}{$1970 \mathrm{~s}$} & \multicolumn{2}{|c|}{$1980 \mathrm{~s}$} & \multicolumn{2}{|c|}{$1990 \mathrm{~s}$} & \multicolumn{2}{|c|}{$2000 \mathrm{~s}$} \\
\hline & Exp & $\operatorname{Imp}$ & Exp & $\operatorname{Imp}$ & Exp & $\operatorname{Imp}$ & Exp & Imp \\
\hline Wheat & 36 & 136 & 40 & 146 & 61 & 162 & 91 & 177 \\
\hline Corn & 58 & 142 & 55 & 149 & 80 & 169 & 102 & 196 \\
\hline Rice & 63 & 175 & 61 & 175 & 90 & 202 & 114 & 219 \\
\hline Sugar & 60 & 165 & 56 & 174 & 81 & 207 & 111 & 222 \\
\hline Beef & 62 & 159 & 64 & 175 & 82 & 202 & 109 & 216 \\
\hline Milk & 48 & 184 & 49 & 186 & 81 & 206 & 116 & 219 \\
\hline Soybean & 30 & 71 & 38 & 91 & 63 & 118 & 87 & 161 \\
\hline
\end{tabular}

Source: Liapis (2012).

Abbreviations: Exp, number of exporters; Imp, number of importers.

In terms of sources of data for the gravity model application, this study employs bilateral trade (import data) from United Nations (UN) Comtrade for the seven commodities noted above. ${ }^{2} \mathrm{UN}$ Comtrade provides data using several nomenclatures, we use the Standard International Trade Classification Revision 1 classification as this classification features data with the longest possible time-frame, that is, from 1962 for some commodities. Specific codes are: 0111 for beef, 0221 and 0222 for milk powder, 041 for wheat, 042 for rice, 044 for corn, 0611 for sugar, and 2214 for soybean. Tariff data are obtained from the UNCTAD Trade Analysis Information System database. For our purposes, we use the simple average for each bilateral trade pair across each of the seven commodities. To account for missing tariff data (countries often only report a single year across a decade or so), the approach of Jayasinghe et al. (2010) is employed to derive implied average tariffs for missing observations. Note that tariff data are only available from 1988.

Data for (35) gravity variables such as GDP, population, contiguity, distance, common language, currency, WTO membership, preferential trade agreements, and colonial ties are from the dynamic gravity dataset constructed by Gurevich and Herman (2018). Their work built a new gravity dataset that improves upon existing resources (e.g., CEPII data) in several ways: first, it was constructed to reflect the dynamic nature of the globe by closely following the ways in which countries and borders have changed between 1948 and 2016. Second, they increased the time and magnitude of variation within several types of variables. All three sources of data are merged to arrive at a data set featuring imports, tariffs, and economic variables from 1988 to $2016 .{ }^{3}$ ML models were trained on various cuts of the data as noted in the next section. The data are in cubical form: country pairs, commodity, and time.

\section{Supervised Machine Learning Model Selection and Validation}

Recall that multiple ML approaches are employed to predict bilateral trade for each of the seven agricultural commodities. Within decision trees, in addition to random forests and extra tree regression, two types of boosting - LightGBM, XGBoost - are considered (Ke et al., 2017):

- LightGBM scans all data instances to estimate the Gain, measured in terms of the reduction in the sum of squared errors, from all the possible Split points. Instead of changing the weights for every incorrectly predicted observation at every iteration like other methods, for example, GBoost, LightGBM tries to fit the new predictor to the errors made by the previous predictor.

\footnotetext{
${ }^{2}$ As pointed out in World Bank (2020), imports are usually recorded with more accuracy than exports because imports generally generate tariff revenues while exports do not.

${ }^{3}$ The economic and commodity data are merged into a SQL database. An R code is used to merge on country-to-country trade transactions, as well as year of economic variables. The data are merged using a SQL Inner Join function, and tariff data were then added computationally using a Python script that matches rows and merges tariff columns.
} 
- LightGBM splits the tree level-wise with the best fit, whereas XGBoost algorithms split the tree leaf-wise. The leaf-wise algorithm can reduce more loss than the level-wise algorithm, and hence can lead to better prediction accuracy.

Using off the shelf Python libraries, each of these models employed all 35 gravity variables noted earlier along with tariffs in the bilateral trade context. Gurevich and Herman (2018) provided 70 gravity variables, but we chose to employ the 35 variables based on correlation analysis. The first step here was to avoid near perfect collinearity among the 70 variables. Then, the data had alternative representations for the size of the economies, for example, GDP total and per capita, and in current and constant dollars. For this second step, measures of feature importance for each of the seven models under alternative variables representing the same phenomenon, for example, size were obtained. The splits and gains then determined the variables to be included in the model. That is, the high cardinality (correlation) of $X$ and alternative representations of $X$ led variable selection by way of information gains from splits (using out-of-the-box python libraries). In most models, only 10-12 features accounted for most of the information gains and they appear consistent with the set of variables employed in traditional econometric analysis. After selecting variables, ML algorithms were deployed. Main parameters tuned for all three boosting methods are maximum depth of the tree, learning rate, number of leaves, and feature fraction. The choice among these supervised models was also dictated by the adjusted R-square, the most commonly used statistical measure in analytics, as shown below (Ke et al., 2017). Both supervised and unsupervised ML methods allow for cross-validation, where predictions can be compared to actuals.

Since data spanned 1962-2016, the training data cut-off point was set at 2010 leaving enough room to compare predictions with actuals starting in 2011. ${ }^{4}$ This partitioning of data allowed for a longer time series to learn as well as have enough data to compare predictions to actuals (2011-2016). Major challenges for many of these algorithms were a large number of zeros in bilateral trade matrices, tariffs as well as time invariant variables in the gravity context such as distance, language, and contiguity. To test the sensitivity of predictions to alternative sets of data, three variations of data were considered to implement ML models: without tariffs (1962-2016), with actual tariffs (1988-2016) and with missing tariffs filled in as per Jayasinghe et al. (2010). Thus, each commodity's trade was subjected to four supervised and a non-supervised model for three different data sets for learning and obtaining predictions for 2011-2016.

Table 2 presents the four best-fitting models among the supervised ML approaches and the training and test sample sizes for each of these models. ${ }^{5}$ The results presented here are from the data set with missing tariffs filled in (1988-2016). While the fit and predictions including validation statistics were similar for the two data sets with tariffs and with missing tariffs filled in, the models using data from 1962 to 2016 yielded lower adjusted R-squares. Some differences in feature importance between these three variations in data were observed, which are detailed in the next section. Note that milk powder had the most observations for training as well as testing among the seven commodities. As can be seen in Table 2, the extra tree regression had the best performance for beef and milk powder, while Random Forest yielded highest $R$-square for corn, rice, and soybean. For sugar, LightGBM provided the best fit. Note, however, adjusted $\mathrm{R}$-squares were similar across the models. The adjusted $R$-square in the bestfitting models ranged between 45 and $83 \%$ (boldfaced numbers in Table 2 ). Lower adjusted $R$-square values for rice and sugar are likely due to high variance in the inputs used for the models and incomplete data from older years. The staple rice, in particular, is often considered a thinly traded commodity with a low share of trade in production for a large number of countries. Both products (rice and sugar) tend to be highly protected by large Asian countries. Under-fitting was not found for any of

\footnotetext{
${ }^{4}$ Gurevich and Herman's (2018) gravity data are not available for 2017 and beyond.

${ }^{5}$ Results from other data set variations (1962-2016 or 1988-2016 without filling in missing tariffs) are not reported in Table 2, but available from authors upon request.
} 
Table 2. Supervised models' validation measures.

\begin{tabular}{|c|c|c|c|c|c|}
\hline Commodity & Observations & LightGBM & XGBoost & Random forest & Extra trees regression \\
\hline Beef & $\begin{array}{l}\text { Training }-27,153 \\
\text { Test }-7,290\end{array}$ & 0.538 & 0.598 & 0.560 & 0.601 \\
\hline Corn & $\begin{array}{l}\text { Training }-29,500 \\
\text { Test }-10,583\end{array}$ & 0.680 & 0.624 & 0.723 & 0.678 \\
\hline Milk Powder & $\begin{array}{l}\text { Training-58,434 } \\
\text { Test-15,594 }\end{array}$ & 0.782 & 0.772 & 0.787 & 0.828 \\
\hline Rice & $\begin{array}{l}\text { Training_-47,697 } \\
\text { Test-12,750 }\end{array}$ & 0.426 & 0.416 & 0.451 & 0.423 \\
\hline Soybean & $\begin{array}{l}\text { Training-22,448 } \\
\text { Test }-6,018\end{array}$ & 0.593 & 0.616 & 0.649 & 0.581 \\
\hline Sugar & $\begin{array}{l}\text { Training }-28,660 \\
\text { Test }-7,644\end{array}$ & 0.448 & 0.347 & 0.439 & 0.447 \\
\hline Wheat & $\begin{array}{l}\text { Training }-26,520 \\
\text { Test }-7,212\end{array}$ & 0.670 & 0.498 & 0.643 & 0.665 \\
\hline
\end{tabular}

the seven commodities and therefore, adjusted $R$-square metric appears to represent the model's quality. Moreover, each of the commodity models was deployed on a global scale, which places all countries on the same level of abstraction when using the response variable. ${ }^{6}$ An additional cut of the data set was also considered: focus on large trading pairs only. While the fit improved considerably when considering large traders only, those results are not reported here given the arbitrary cut to the sample data.

\section{Deep Neural Networks Model}

Neural networks, a form of deep learning, can take several paradigms: recurrent and convolutional neural networks, hybrids, and Multilayer Perceptron (MLP). This study employs MLP given the tabular data context (along with its cardinality) and the goal of obtaining better predictions. MLP is a feed-forward algorithm with the following steps:

- \#1, Forward pass: here, the values of the chosen variable are multiplied with weights and bias is added at every layer to find the calculated output of the model.

- \#2: Calculate the error or the loss: the data instance is passed, the output is called the predicted output; and that is compared with real data called the expected output. Based upon these two outputs, the loss is calculated (using Back-propagation algorithm).

- \#3: Backward pass: the weights of the perceptrons are updated according to the loss.

In Python, the MLP algorithm uses Stochastic Gradient Descent (SGD) for the loss function (Scikit, 2019). SGD is an iterative method for optimizing an objective function with smoothness properties. ${ }^{7}$ As noted earlier, neural networks do not have obvious validation statistics, unlike supervised models.

\footnotetext{
${ }^{6}$ Zeros were used to fill missing bilateral trade data for all commodities. This can lead to the oversampling of zeros in these datasets. Oversampling increases the count of minority class instances to match it with the count of majority class instances, that is, "upsizing" the minority class. Additional exploration of undersampling or correlation imputations for example could improve the models results, a topic for further research.

${ }^{7}$ For the MLP method, the MLPClassifier function is used along with the following parameters: early_stopping, epsilon, hidden_layer_sizes, learning_rate, learning_rate_init, max_iter, momentum, power_t, validation_fraction.
} 


\section{Results and Discussion}

In addition to employing ML techniques, this study considered traditional approaches to estimating the gravity Equation (7). As noted by Disdier and Head (2008), many econometric techniques have been used in estimating Equation (7), but the more recent approach that accounts for zeros in $Y_{i j t}$, heteroscedasticity in additive errors to Equation (7) and other issues is PPML estimation (Santos Silva and Tenreyro, 2006). The PPML approach and its variations in the high-dimension context, posed several specification and estimation challenges. In particular, the pair-wise fixed effects, for example, origin-time and destination time, as in Yotov et al. (2016) as well as Correia et al. (2019), created significant collinearity issues and convergence problems. Nonetheless, results from estimating Equation (7) using basic PPML methods and the chosen 35 gravity variables (plus tariffs) for the seven commodities of this study are reported in the Appendix I. Given the limited ability of PPML-based methods to identify the relative importance of explanatory variables (in the presence of thousands of fixed effects) as noted in the World Economic Outlook, IMF (2019), the following section presents results from ML methods only. ${ }^{8}$

Figures $1-3$ and Tables 3 and 4 present the results from the supervised learning models, while Figure 4 details those from the neural networks application to capturing the gravity trade relationship in Equation (7).

\subsection{Supervised ML model results}

Recall that the cut-off year for the training data was 2010 and so, projections from the supervised models are made through 2016. The seven panels of Figure 1 show the 2011-2016 aggregate (total) trade values, actual and predicted, for each of the chosen commodities. We present the predicted values from the best-fitting model (bold-faced $R$-square in Table 2) in Figures 1-3. ${ }^{9}$ Multiple factors contribute to the fit, but chief among them are the pruning of the decision trees, data incompleteness across commodities (training versus test data size), cardinality of the predictors and boosting to improve the successive fit of decision trees. For corn, milk powder, rice, sugar, and wheat, the predictions in Figure 1 track the general pattern of actual trade values from 2011 to 2014. Note from Table 2 that corn, milk powder, and wheat have some of the highest adjusted $R$-squares, while milk powder and rice have the highest number of observations for training and testing. While the gap between the actual and predicted can be attributed to the fit, the model fit itself is likely influenced by the number and quality of underlying data as noted above. Predictions of aggregate trade values for soybeans and beef deviate from the pattern of actuals during 2011-2014. Note that the soybean models have the fewest observations available for training, partly due to the delayed expansion in the number of countries trading soybeans as shown in Table $1 .{ }^{10}$

Note, however, all models' predictive ability considerably deteriorates for 2015-2016. A closer examination of actuals and predicted values for each bilateral pair indicates that the zero values, prevalent in the gravity models, are at the core of the falling predictive abilities of supervised ML in 2015-2016 (Figure 1). For instance, the zero values of trade data are often initially augmented by boosting or extra trees to be a small positive or negative number, which upon iteration expands further to widen the gap between actuals and predictions. Thus, the above results suggest that boosting techniques might be better at near- to medium-term projections relative to those over a longer horizon. The patterns seen in Figure 1 are unlike the straight-line projections commonly observed in aggregate trade projections by WTO (2019) and OECD (2019), and agricultural trade projections of USDA (2019). Current projections by major international and national agencies are a combination of expert-judgment and time-series analysis.

\footnotetext{
${ }^{8}$ As noted earlier, a direct comparison of gravity results can only be made with prescriptive ML models, which are under further development.

${ }^{9}$ Figures 1-3 for each of the ML models (LightGBM, XGBoost, Random Forests, and Extra Tree Regression) showed variations depending on the fit, but the general pattern described here fits all of them.

${ }^{10}$ Note that for other commodities more hyper parameters can be tuned further to achieve high predictive accuracy, but results are presented as such to highlight the advantages and disadvantages of alternative ML approaches.
} 
Beef

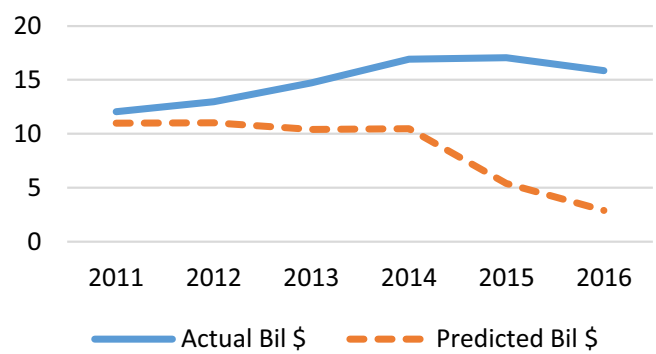

Milk Powder

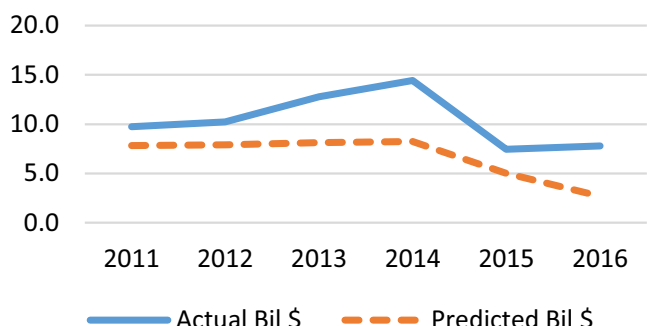

Soybean

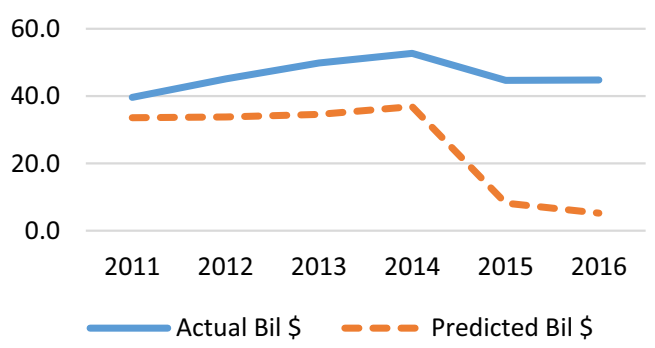

Corn

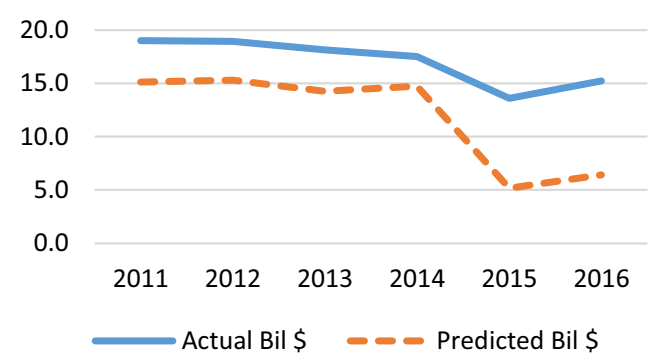

Rice

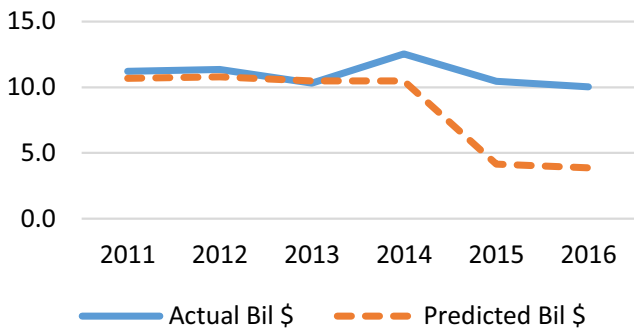

Sugar

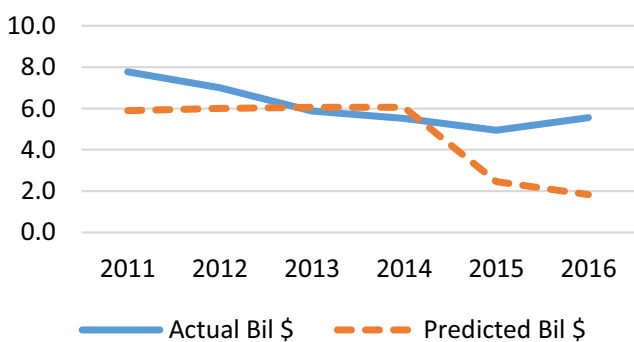

\section{Wheat}

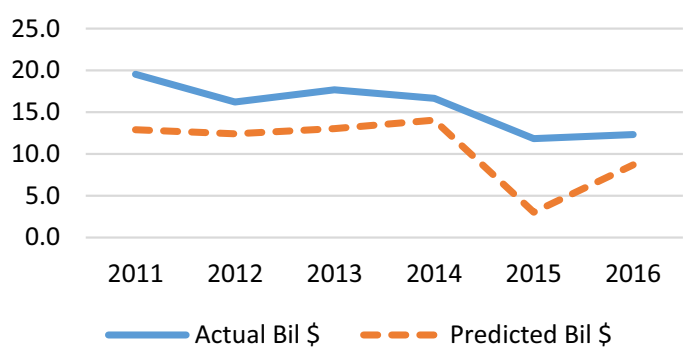

Figure 1. Supervised model predictions of aggregate trade values, 2011-2016.

As noted earlier, such projections have low forecast accuracy, for example, USDA (2019) at 35\%, or have limited explanatory power (World Economic Outlook IMF, 2019).

Figures 2 and 3 present predictions for the two major bilateral pairs, in terms of trade value, for each of the seven commodities. Seven out of the 12 pairs shown in Figures 2 and 3 have predictions closely tracking actuals: corn (USA-Japan; USA-Mexico), rice (India-Saudi Arabia; USA-Mexico), wheat 
Beef: Australia-Japan

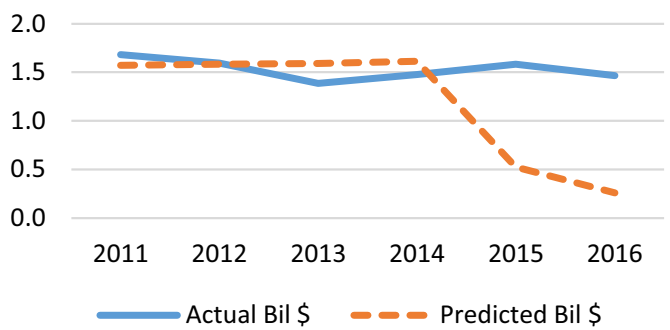

Milk Powder: New Zealand- China

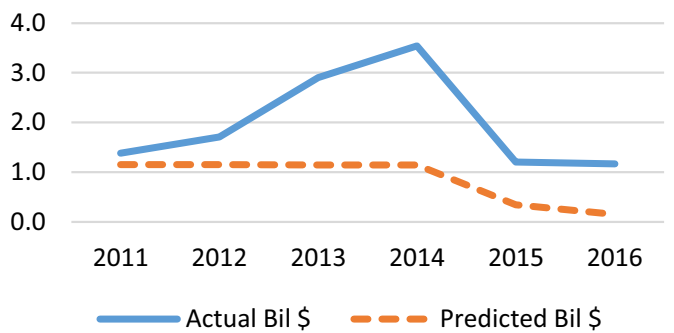

Soybean: Brazil - China

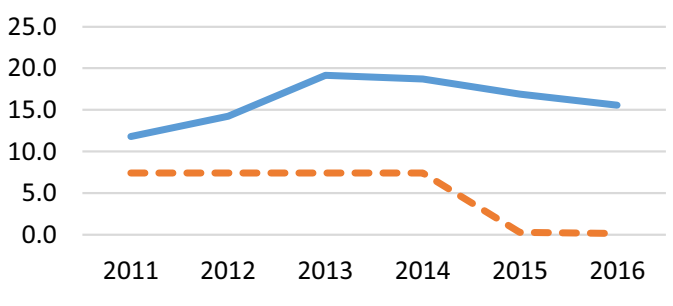

Actual Bil \$ _ - Predicted Bil \$
Corn: U.S. - Japan

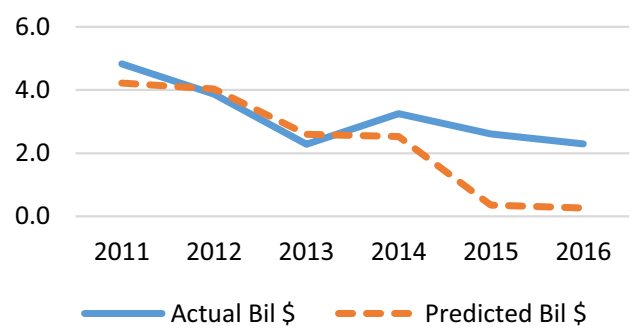

Rice: India - Saudi Arabia

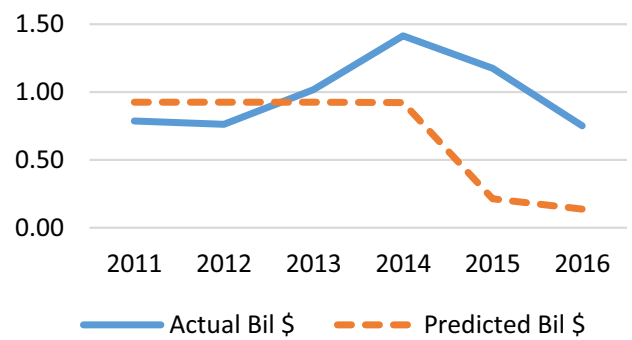

Sugar: Brazil - China

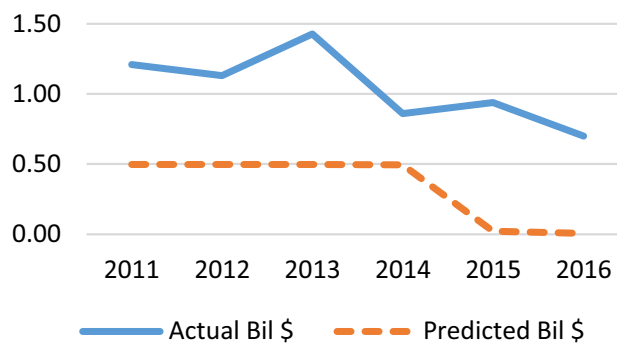

Wheat: U.S. - Japan

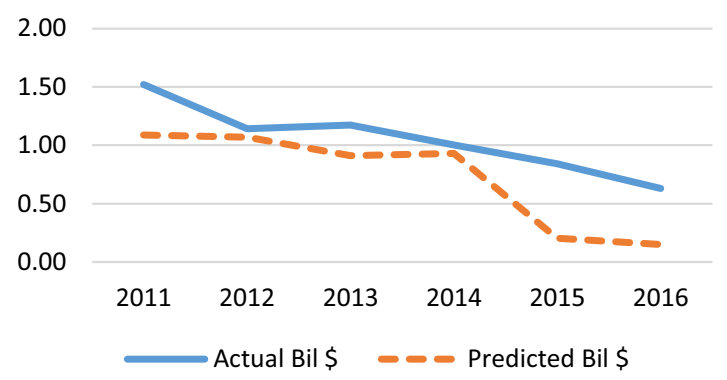

Figure 2. Supervised model predictions of top partners' trade values, 2011-2016.

(USA-Japan), and beef(Australia-Japan; USA-Mexico) also have bilateral predictions that closely track actuals for 2011-2014. As noted earlier, the soybean model had data quality issues, and both sugar and soybean projections improved when estimated with a sample containing large countries only. Recall that each of the models is deployed on a global scale, but as observed in the data and noted by Anderson (2016), agricultural commodity trade is concentrated in few countries in the early years of the sample. 
Beef: U.S.-Mexico

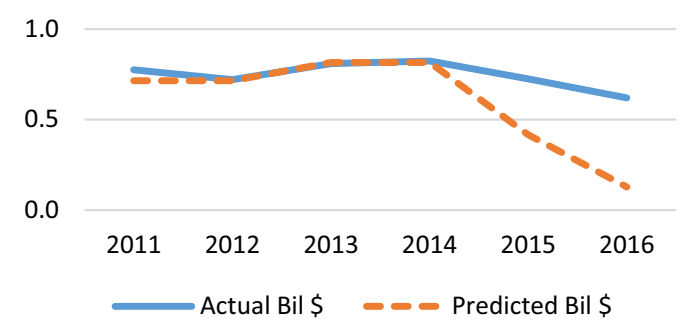

Milk Powder: U.S.-Mexico

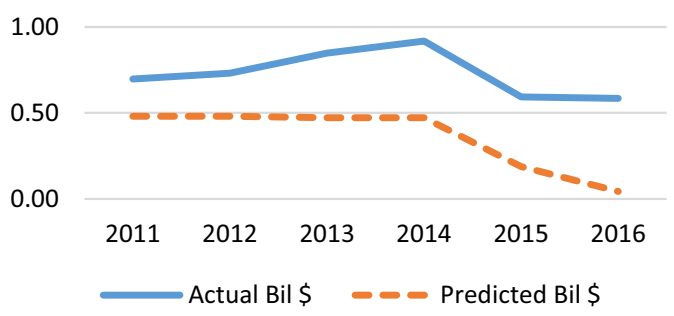

Soybean: U.S. - China

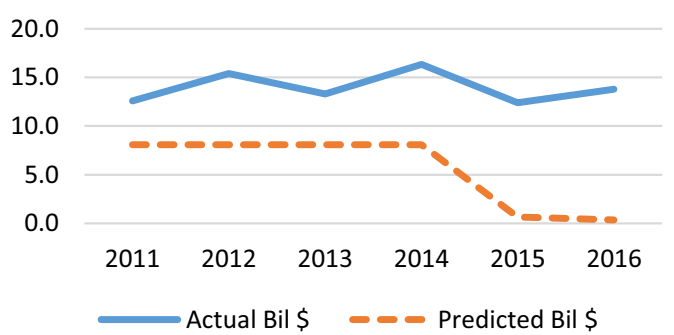

Corn: U.S. - Mexico

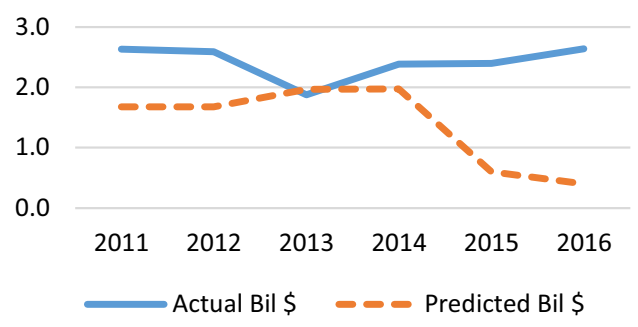

Rice: U.S. - Mexico

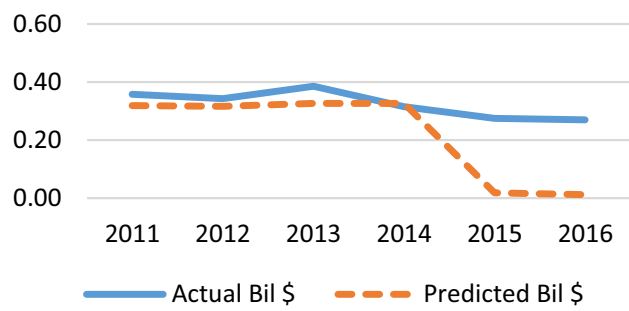

Sugar: Thailand - Japan

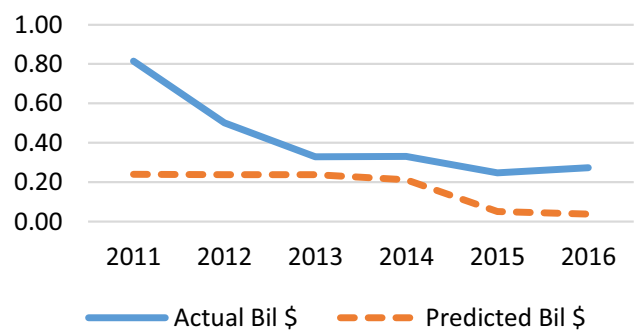

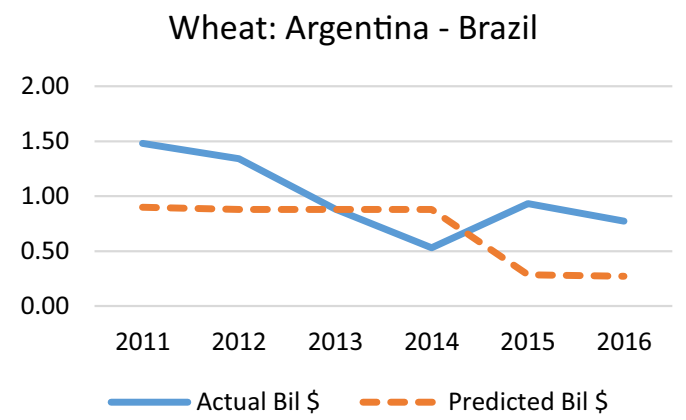

Figure 3. Supervised model predictions of 2nd top partners'trade values, 2011-2016.

Grouping or categorizing countries and hyper-tuning variables yielded better model fits, but valuable observations are lost by employing arbitrary cut-offs. As with Figure 1, for all commodities, the deterioration of predictive ability during 2015-2016 is attributable to zero trade values and the associated extra trees or boosting.

We compare supervised ML with neural networks models later, but an advantage of the former is its ability to identify from among the set of predictors the features that have the greatest importance. Decision 
Table 3. Ranking variables by information gain (normalized values).

\begin{tabular}{lccccccc}
\hline Variables & Beef & Corn & Milk powder & Rice & Soybean & Sugar & Wheat \\
\hline Population_Origin & 1 & 1 & 1 & 1 & 4 & 4 & 1 \\
Population_Destination & 3 & 7 & 6 & 2 & 3 & 1 & 7 \\
Distance & 9 & 8 & 3 & 3 & 2 & 3 & 6 \\
GDP Per Capita_Origin & 5 & 2 & 8 & 4 & 8 & 9 & 5 \\
Longitude_Destination & 4 & 5 & 10 & 11 & 1 & 5 & 4 \\
Latitude_Destination & 6 & 4 & 4 & 7 & 9 & 2 & 9 \\
Longitude_Origin & 7 & 6 & 2 & 8 & 6 & 11 & 3 \\
Latitude_Origin & 2 & 3 & 9 & 6 & 11 & 10 & 2 \\
GDP PerCapita_Destination & 8 & 9 & 7 & 10 & 7 & 6 & 8 \\
Time & 11 & 10 & 5 & 9 & 5 & 8 & 10 \\
Tariffs & 10 & 11 & 11 & 5 & 10 & 7 & 11 \\
\hline
\end{tabular}

Table 4. Relative importance of variables based on information gain (percent).

\begin{tabular}{lccccccc}
\hline Variables & Beef & Corn & Milk powder & Rice & Soybean & Sugar & Wheat \\
\hline Population_Origin & 100 & 100 & 100 & 100 & 67 & 43 & 100 \\
Population_Destination & 78 & 9 & 48 & 80 & 84 & 100 & 30 \\
Distance & 9 & 8 & 61 & 69 & 89 & 70 & 33 \\
GDP Per Capita_Origin & 55 & 90 & 34 & 63 & 30 & 18 & 40 \\
Longitude_Destination & 66 & 15 & 20 & 26 & 100 & 39 & 44 \\
Latitude_Destination & 53 & 16 & 54 & 41 & 14 & 92 & 17 \\
Longitude_Origin & 34 & 12 & 81 & 35 & 39 & 9 & 48 \\
Latitude_Origin & 92 & 39 & 34 & 49 & 5 & 17 & 72 \\
GDP Per Capita_Destination & 27 & 6 & 36 & 31 & 30 & 29 & 27 \\
Time & 10 & 5 & 53 & 33 & 55 & 19 & 17 \\
Tariffs & 15 & 2 & 13 & 52 & 6 & 24 & 9 \\
\hline
\end{tabular}

Notes. 100 indicates the variable with the highest information gain. All other variables together accounted for another $84-126 \%$ of information gains across the seven commodities.

tree methods, particularly in the context of boosting, randomize among predictors for splitting the tree into nodes (or leafs) and the repetitive process searches for predictors and cut-off values that offer the greatest decline in the total-sample sum of squared errors (Equation (5)). In doing so, these methods identify the information gain, that is, the reduction in total-sample sum of squared residuals, from each of the predictors. Recall that the gravity model, Equation (7), in this study employed 35 variables plus tariffs as predictors. Tables 3 and 4 show both the ranking of predictors by information gain and their relative importance, respectively.

\subsection{Economic significance in supervised ML models}

Table 3 presents the top 11 variables which provided the largest information gains from the 36 predictors included in these models. Alternatively termed as feature importance, they point to which of the variables are most indicative of the response variable (bilateral trade) in the model. Note that removing any of these top predictors would drastically change the model results. Across commodities, the top 11 predictors remained the same, but their ranking and relative importance was different. As a review of gravity models predict, the size of the two economies engaged in trade has the largest influence in reducing the 
Australia - Beef Exports

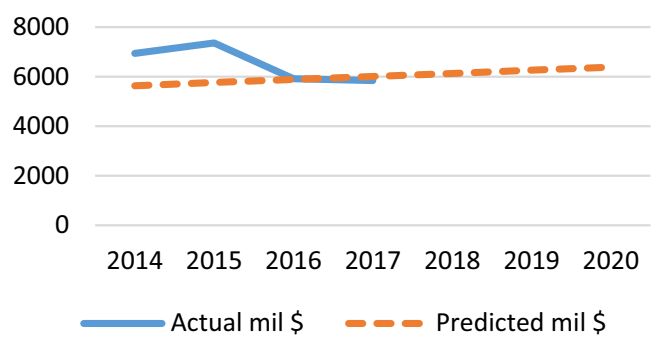

US - Rice Exports

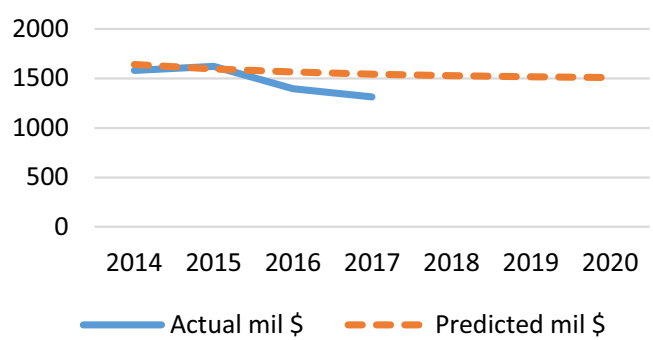

Australia - Sugar Exports

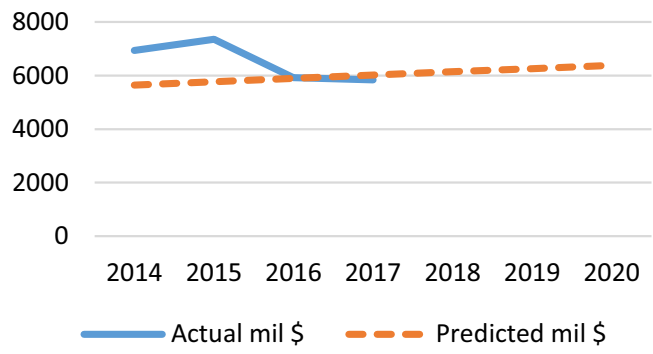

US - Corn Exports

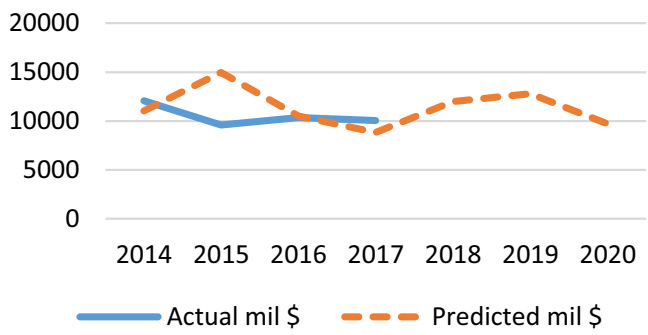

US - Soybean Exports

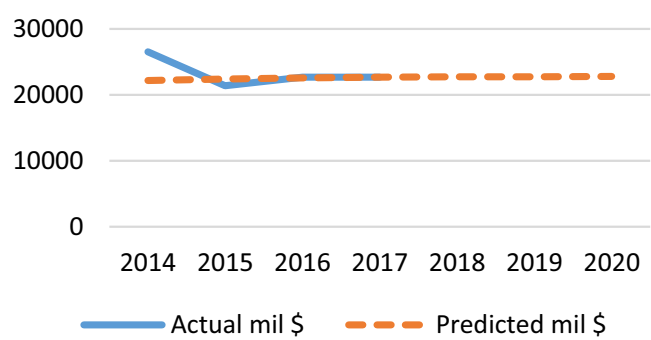

Netherlands - Milk Powder Exports

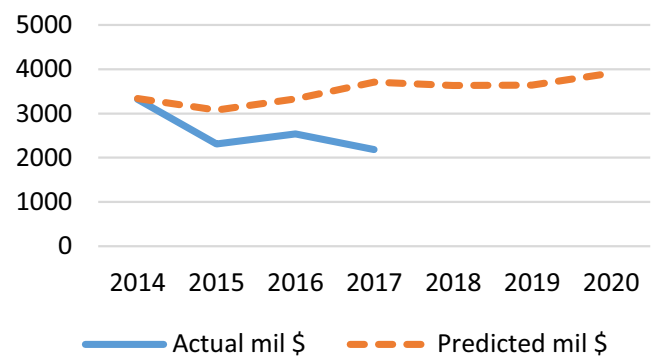

\section{US - Wheat Exports}

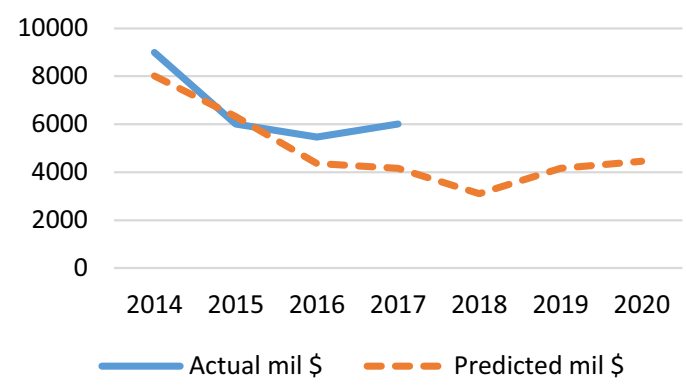

Figure 4. Neural networks prediction of top country's aggregate exports, 2014-2020.

total-sample sum of squared errors. So, the population of origin and destination are, on average, the top 2 information providers in the learning of agricultural trade flows. This result is largely consistent with commodity trade, which is significantly determined by the importing country's size. Note that the origin country's size also matters for all the reasons noted in gravity models, that is, large countries tend to trade more with other large countries (Yotov et al., 2016; Chapter 4, World Economic Outlook-International 
Monetary Fund, 2019). Distance is the next predictor offering substantial information gains in the learning and prediction of trade flows. There is some variation in the ranking of distance among commodities, as with the population of destination, ranging from 2 in soybean to 9 in the case of beef. In gravity models, latitude and longitude are often employed to represent the remoteness of a country (spatially and temporally). ${ }^{11}$ The supervised models indicate the high relevance of both latitude and longitude of both origin and destination to predict commodity trade flows. Time-specific effects and tariffs were respectively 10th and 11th indicators of information gain. Note that tariffs are relatively more important in the case of rice and sugar, two of the highly protected agricultural commodities across countries.

The rankings in Table 3 confirm the significance of commonly used gravity predictors but do not completely capture the relative importance of these variables. Table 4 normalizes the information gain of each variable with that of the top predictor (ranked \#1 in Table 3) for each commodity. Similar to Table 3, most information gains in the supervised learning models arise from the size of the two economies. The variation in the gain associated with the distance, latitude/longitude, and time predictors across commodities likely capture not only policy-induced differences but also influences of extreme events associated with specific time periods (e.g., droughts or floods affecting particular growing regions). As in Table 3, tariffs are relatively more important providers of information in the case of rice and sugar. An interesting aspect of these gains is the likelihood that they vary by the size of the training sample, which indicates that the impact of distance or any other feature can vary over sub-samples, offering a potential solution to some puzzles, for example, the distance puzzle, commonly observed in econometric estimation of gravity models (Yotov et al., 2016). Together, the remaining 25 variables accounted for $84-126 \%$ of information gains relative to the top predictor for each commodity. Factors such as common language or border, FTA or WTO membership, and others matter collectively, but each of their effects is not large relative to the economies' size or distance between trade partners.

\subsection{Deep neural networks model results}

Turning now to neural networks, Figure 4 presents predictions for the top exporter for each of the seven commodities. Recall from the discussion of Equations (6a) and (6b) that these models do not necessarily have a response variable. In that sense, learning here happens primarily with the bilateral trade data. Python libraries are used to deploy the neural network, namely, scikit-neuralnetwork. The training sample cut-off is set at 2014 and projections are made until 2020. Figure 4 shows that all commodities' predictions, with the exception of Netherlands' milk powder exports, closely track respective actuals for 2014-2017. In fact, there is at least one projection that almost mimics actual in all projections except in the case of milk powder.

It is not straightforward to compare ML and deep learning models, but each has its advantages and disadvantages (Storm et al., 2019). Likewise, comparisons to traditional/econometric approaches are tenuous given causality issues noted earlier. Nonetheless, an attempt is made here to explore the merits of each approach and relevance in specific contexts. The supervised machine learning models, primarily of the decision-tree kind with bagging, random forests, or boosting, have a structure relating a response variable to set of predictors. The estimated structure is often heterogeneous, non-linear, and based on repeated learning, that is, minimizing the total-sample sum of squared errors. The supervised techniques are straight forward to implement particularly in uncovering complex relationships and can be compared among themselves in terms of validation statistics such as error sum of squares. They also provide information on the most relevant predictors including the relative strength of alternative predictors. However, this technique cannot provide standard errors on predictors' contribution or a coefficient capturing the relationship between $\boldsymbol{Y}$ and $\boldsymbol{X}$, due in part to the non-linear and repeated learning process noted above. These models are a great fit for problems primarily focused on near- to medium-term predictions, for example, prices, trade flows, especially when a large volume of data are available. Neural networks carry similar advantages in prediction problems, but do not have predictors. As demonstrated

\footnotetext{
${ }^{11}$ As Anderson (2014) notes, longitude matters for trade by capturing time and communication differences. In other instances, remoteness has often been used to capture multilateral resistance in gravity models (Yotov et al., 2016).
} 


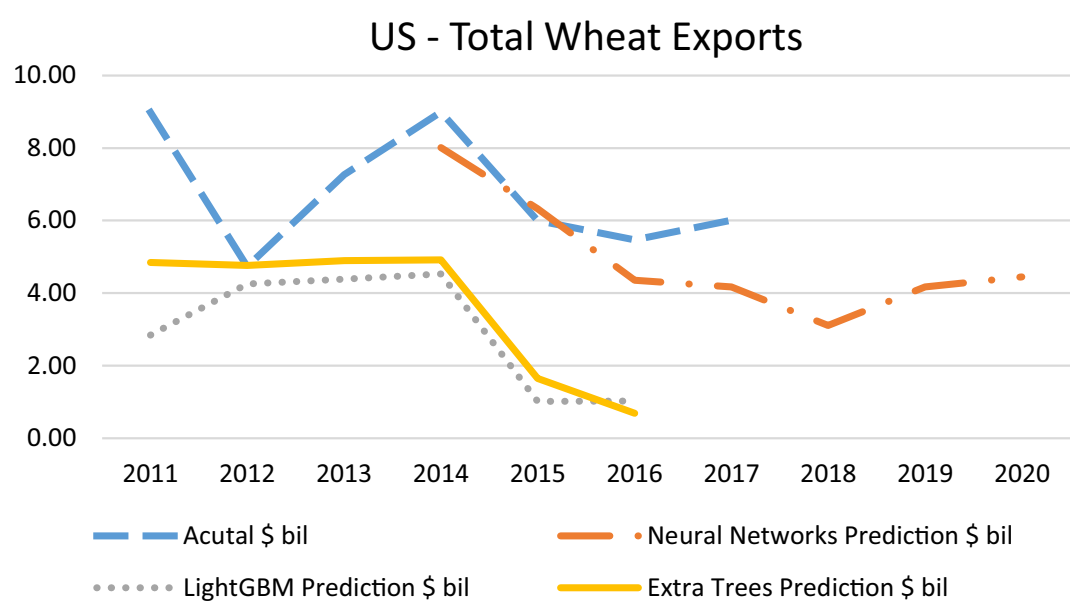

Figure 5. Comparison of predictions from supervised models and neural networks.

using bilateral trade data predictions, they appear most suited to longer-term projections, for example, climate change. Figure 5 confirms this claim using the example of U.S. wheat exports to all countries, that is, actuals and predictions from three models - LightGBM, Extra trees regression, and neural networksshow the better long-term fit of neural networks. Note that supervised techniques with rolling training samples can also generate a sequence of medium-term projections that can be integrated for longer-term projections. ${ }^{12}$ The pruning and regularization involved in supervised methods may limit the amount of data used in learning (as shown in Table 2 on training and test data), whereas neural network approaches attempt to use all data. In contrast, econometric approaches offer structure and inference with carefully chosen causal relationships, often linear and homogeneous. The later are seldom cross-validated and also suffer from specification or variable selection bias like (unlike) supervised (unsupervised) models.

\section{Outlier Events (Future Work) and Conclusions}

\subsection{Outlier events and trade}

We draw conclusions from the study in Section 7.2, but point to an interesting extension in this section given the ongoing pandemic: how to generate predictions during an outlier event? The simple answer is to inject "context" into predictions and provide solutions during outlier events.

Deploying context to represent outlier events is not a straight forward task. The context within a dataset can be represented as features (Turney, 2002). Features in general fall into three categories: primary features, irrelevant features, and contextual features. Primary features are the traditional ones which are pertinent to a particular domain. Irrelevant features are features which are not helpful and can be safely removed, while contextual features are the ones to pay attention to. That categorization eliminates irrelevant data but is of little help in clearly defining context. A promising method to solve this challenge is the Recognition and Exploitation of Contextual Clues via Incremental Meta-Learning (Widmer, 1996), which is a two-level learning model in which a Bayesian classifier is used for context classification, and meta algorithms are used to detect contextual changes. An alternative is the context-sensitive feature selection (Domingos, 1997), a process that out performs traditional feature selection such as forward and backward sequential selection. Dominogos's method uses a clustering-based approach to select locally relevant features. Additionally, Bergadano et al., (1992) introduced a two-tier contextual classification adjustment method called POSEIDON. The first tier captures the basic properties of context, and the

\footnotetext{
${ }^{12}$ For example, changing the training data cut-off to 2009 or 2011 would likely generate better projections for 2010-2013 and 2012-2015, respectively.
} 
second tier captures property modifications and context dependencies. Context injections, however, have been more successful when they are applied to specific domains (none to date on agricultural economics or trade). For example, adding context to data yielded significant improvements in time and quality of software testing (Batarseh, 2014).

The issue of deriving context from data however is even more challenging. For instance, Williams (2018) pointed out that data science algorithms without realizing their context could have an opacity problem. This can cause models to be racist or sexist, for example, a word embedding algorithm classified European names as pleasant and African American names as unpleasant (Zou et al., 2018). If a reductionist approach is considered, adding or removing data can surely redefine context, especially in the case of outlier events. It is observed, however, that most real-world data science projects use incomplete data (Kang, 2013; Sesa and Syed, 2016). Data incompleteness occurs within one of the following categorizations: (a) Missing Completely at Random (MCAR), (b) Missing at Random (MAR), and (c) Missing not at Random. MAR depends on the observed data, but not on unobserved data while MCAR depends neither on observed data nor unobserved data (Schafer and Graham, 2002; Graham, 2009). There are various methods to handle missing data issues which includes listwise or pairwise detections, multiple imputation, mean $/ \mathrm{median} /$ mode imputation, regression imputation, as well as learning without handling missing data.

\section{2. $M L$ for trade}

This study introduced ML models to the international trade setting and posed questions on their applicability and prediction quality. The basic specification of the popular gravity model of trade flows was subjected to machine and deep learning processes using data from 1962 to 2016 . A loss function that summed the squared error between actual and predicted bilateral trade flows for all available country pairs was minimized with supervised ML models including decision trees such as random forests, bagging, and alternative types of boosting. Supervised ML models employed a set of predictors, commonly used gravity variables such as size of economies, the distance between them, and associated frictions. The validation statistics along with the data properties (distribution, cardinality, and completeness) helped explain predictions and the relative importance of gravity variables from supervised ML models. Neural networks are also employed in this study to uncover relationships of a single variable (trade) without the need for predictors (gravity variables). Both models are cross-validated, that is, in ML models the training data were set to 1962-2010 and the testing data to 2011-2016, while neural networks model's training and test data were set to 1962-2014 and 2015-2020, respectively.

Results from supervised ML show that the models fit well in the near to medium term (2011-2014), that is, predictions closely track actuals, when the models have a high-adjusted $R$-square and trade data encompass a large number of countries and years. However, the large presence of zero trade values and the use of extra trees or boosting to transform weak supervised learners into strong ones cause predictive quality to fall 3-4 years from the training data cut-off. A major advantage of the supervised ML model is the ability to identify which variables among the set of predictors provides more information to understanding bilateral trade flows. A ranking of top 11 variables by information gain, that is, reduction in the total-sample sum of squared errors of the loss function attributable to a predictor, and their relative importance show that economies size, distance between them, location of countries, time, and tariffs are more important than other gravity variables in explaining trade flows. While these results are consistent with the trade and gravity model literature, ML's strengths are in variable selection, prediction, and economic significance. In addition, the supervised ML models have opened up an opportunity to address time- and space-varying effects, for example, the distance puzzle. Varying the training sample size likely yields different contributions by features, but other model criteria need to be carefully considered to fully unlock such puzzles. Deep learning models (i.e., neural networks) appear to be better suited for long-term forecasting with predictions closely tracking actuals across commodities. However, they are known to be black boxes and are often difficult to validate.

Predicting agricultural trade patterns is critical to decision making in the public and private domains, especially in the current context of trade wars with tit-for-tat tariffs. For instance, farmers likely consider 
the potential demand from alternative foreign sources before deciding to plant crops, especially in large exporters. Similarly, countries setting budgets for farm programs need better predictions of prices and trade flows for assessing domestic production and consumption needs and instruments employed to achieve those outcomes. This study demonstrates the high relevance of ML models to predicting trade patterns with a greater accuracy than traditional approaches for a range of time periods. Existing forecasts of trade such as those by WTO, OECD, and USDA are a combination of model-based analyses and expert judgment and tend to have high variability. A comparison of ML to PPML-based methods is hindered by the latter's specification, convergence, and collinearity challenges, and limited ability to identify the relative importance of explanatory variables. The ML models, by relying on data and deep learning, allow for alternative and robust specifications of complex economic relationships. Moreover, the ML models are cross-validated and provide ways to simulate trade outcomes under alternative policy scenarios including their uncertainty in recent times. Like computable general equilibrium models, which are popular in assessing the effects of trade policy changes, ML can aid in modeling alternative policy scenarios. For instance, varying training or testing data with prohibitive tariffs or trade bans can provide predictions which can be compared with traditional models. Future work focusing on other industries, for example, manufacturing, data/matrix completeness (a major issue when dealing with zeros in trade and tariffs), multi-variate response variables and prescriptive ML techniques to compare with current causal models would greatly aid in public and private decision making.

Funding Statement. This work received no specific grant from any funding agency, commercial, or not-for-profit sectors.

Competing Interests. The authors declare no competing interests exist.

Data Availability Statement. Code samples and examples, as well as data sets are available under this GitHub repository: https:// github.com/ferasbatarseh/TradeAI

Author Contributions. Conceptualization, M.G.; Methodology, F.B. and M.G.; Formal analysis F.B., M.G., J.B., A.K., and S.J.; Data curation, F.B., A.K., and S.J.; Writing—original draft, M.G., Writing-review and editing, M.G., J.B., S.J., and F.B.; Supervision, M.G.

Disclaimer. The findings and conclusions in this paper are those of the authors and should not be construed to represent any official USDA or U.S. Government determination or policy.

Supplementary Materials. To view supplementary material for this article, please visit http://dx.doi.org/10.1017/dap.2020.22.

\section{References}

Anderson JE (1979) A theoretical foundation for the gravity equation. American Economic Review 69(1), $106-116$.

Anderson E (2014) Time differences, communication and trade: longitude matters. Review of World Economics 150(2), 337-369.

Anderson K(2016) Agricultural trade, policy reforms, and global food security. In Palgrave Studies in Agricultural Economics and Food Policy. New York: Palgrave MacMillan, pp. 61-83.

Anderson JE and van Wincoop E (2003) Gravity with gravitas: a solution to the border puzzle. American Economic Review 93(1), $170-192$

Athey S and Imbens GW (2019) Machine Learning Methods Economists Should Know About. Working Paper, Palo Alto, CA: Stanford University.

Bajari P, Nekipelov D, Ryan SP and Yang M (2015) Machine learning methods for demand estimation. American Economic Review 105(5), 481-485.

Batarseh FA (2014). Context-driven testing on the cloud. In Context in Computing. New York, NY: Springer, pp. 25-44.

Batarseh F and Yang R (2017) Federal Data Science: Transforming Government and Agricultural Policy Using Artificial Intelligence. New York: Elsevier's Academic Press.

Baxter M and Hersh J (2017). Robust Determinants of Bilateral Trade. Paper Presented at Society for Economic Dynamics.

Bergadano F, Matwin S, Michalski RS and Zhang J (1992). Learning two-tiered descriptions of flexible concepts: the POSEIDON system. Machine Learning 8(1), 5-43.

Correia S, Guimaraes P and Zylkin T (2019) PPMLHDFE: Fast Poisson Estimation with High-Dimensional Fixed Effects. Available at https://arxiv.org/abs/1903.01690v3 (accessed 18 March 2020).

Disdier AC and Head K (2008) The puzzling persistence of the distance effect on bilateral trade. Review of Economics and Statistics 90(1), 37-48.

Domingos P (1997). Context-sensitive feature selection for lazy learners. In Lazy Learning. Dordrecht: Springer, pp. $227-253$. 
Feenstra RC (2004) Advanced International Trade: Theory and Evidence. Princeton, NJ: Princeton University Press.

Graham JW (2009). Missing data analysis: making it work in the real world. Annual Review of Psychology 60, 549-576.

Gurevich T and Herman P (2018) The Dynamic Gravity Dataset: Technical Documentation. Washington, DC: U.S. International Trade Commission.

James G, Witten D, Hastie T and Tibshirani R (2013). An Introduction to Statistical Learning. New York: Springer.

Jayasinghe S, Beghin JC and Moschini GC (2010). Determinants of world demand for U.S. corn seeds: the role of trade costs. American Journal of Agricultural Economics 92(4), 999-1010.

Kang H (2013). The prevention and handling of the missing data. Korean Journal of Anesthesiology 64(5), pp. $402-406$.

Ke G, Meng Q, Finley T, Wang T, Chen W, Ma W, Ye Q and Liu TY (2017) LightGBM: A Highly Efficient Gradient Boosting. In 31st Conference on Neural Information Processing Systems, NIPS. Long Beach, CA, pp. 1-9.

Liapis P (2012) Structural Change in Commodity Markets: Have Agricultural Markets Become Thinner? Food, Agriculture and Fisheries Paper No. 54. Paris: Organization for Economic Cooperation and Development.

Mullainathan S and Spiess J (2017) Machine learning: an applied econometric approach. Journal of Economic Perspectives 31(2), $87-106$.

Organization for Economic Cooperation and Development, Trade in Goods and Services Forecast. https://doi.org/10.1787/ 0529d9fc-en (accessed 30 September 2019).

Santos Silva JM and Tenreyro S (2006) The log of gravity. Review of Economics and Statistics 88(4), 641-658.

Schafer JL and Graham JW (2002). Missing data: our view of the state of the art. Psychological Methods 7(2), 147.

Scikit Available at https://scikit-learn.org/stable/modules/sgd.html (accessed 30 September 2019).

Sessa J and Syed D (2016). Techniques to Deal With Missing Data. In Electronic Devices, Systems and Applications (ICEDSA) 5th International Conference, pp. 1-4.

Storm H, Baylis K and Heckelei T (2019) Machine learning in agricultural and applied economics. European Review of Agricultural Economics 47(3), 849-892. https://doi.org/10.1093/erae/jbz033

Turney PD (2002). The Management of Context-Sensitive Features: A Review of Strategies. In 13th International Conference on Machine Learning, Workshop on Learning in Context-Sensitive Domains, Bari, Italy, pp. 60-66.

U.S. Department of Agriculture (2019). Outlook for U.S. Agricultural Trade. Washington, DC: Economic Research Service. Available at https://www.ers.usda.gov/publications/pub-details/?pubid=94836 (accessed 30 September 2019).

Varian H (2014). Big data: new tricks for econometrics. Journal of Economic Perspectives 28(2), 3-28.

Widmer G (1996). Recognition and Exploitation of Contextual Clues Via Incremental Meta-Learning (Extended Version). In The 13th International Conference on Machine Learning. San Francisco: Morgan Kaufmann, pp. 525-533.

Williams MA (2018). Risky Bias in Artificial Intelligence. The Australian Academy of Technology and Engineering. Available at https://www.atse.org.au/content/news/risky-bias-in-artificial-intelligence.aspx (accessed 27 August 2020).

World Bank. Mirror Data with UN COMTRADE. Available at https://wits.worldbank.org/wits/wits/witshelp/Content/Data Retrieval/T/Intro/B2.Imports_Exports_and_Mirror.htm (accessed 18 March 2020).

World Economic Outlook (2019). Washington DC: International Monetary Fund.

World Trade Organization. Available at https://www.wto.org/english/news_e/pres19_e/pr837_e.htm (accessed 30 September 2019).

Yotov Y, Piermartini R, Monteiro JA and Larch M (2016). An Advanced Guide to Trade Policy Analysis: The Structural Gravity Model. Geneva: World Trade Organization.

Zou J and Schiebinger L (2018). AI Can Be Sexist and Racist-It's Time to Make It Fair. Available at https://www.nature.com/ articles/d41586-018-05707-8 (accessed 27 August 2020).

\section{Appendix I: Poisson Pseudo Maximum Likelihood Estimation of the Gravity Model}

Examining factors that determine trade patterns has largely been accomplished through the use of the gravity model—described as the workhorse of international trade and one of the most successful empirical models in economics (Anderson and van Wincoop, 2003; Yotov et al., 2016). The gravity model has been developed over the years to incorporate different explanatory variables depending on the questions to be answered, and to address some of the empirical issues such as the large amount of zeros in trade flows. In particular, the Poisson Pseudo Maximum Likelihood (PPML) laid out in Santos Silva and Tenreyro (2006) allows for the inclusion of zero trade flows and corrects for heteroscedasticity which often plague estimation of the gravity model. Additionally, the importance of accounting for observable and unobservable country-level heterogeneity and multilateral resistance terms through the use individual and pair-wise fixed effects (Feenstra, 2004).

To complement the ML application, the PPML regression considered below included imports in levels as the dependent variable, while the non-dummy independent variables are specified in log terms. Given problems in variable selection, the ML application was used as the basis for the following specification (Yotov et al., 2016):

$$
\begin{aligned}
v_{i j}^{k}= & \exp \left[\alpha_{i}+b_{j}+G D P_{i}+G D P_{j}+\text { Pop }_{i}+\text { Pop }_{j}+\beta_{1} \text { distance }_{i j}\right. \\
& +\beta_{2} \text { time }+\beta_{3} \text { longitude }_{j}+\beta_{4} \text { latitude }_{j}+\beta_{5} \text { poltical }_{j},
\end{aligned}
$$


Table A1. Results from PPML estimation of the gravity model.

\begin{tabular}{|c|c|c|c|c|c|c|c|}
\hline & Beef & Corn & Milk & Rice & Soy & Sugar & Wheat \\
\hline $\operatorname{gdp}_{\mathrm{x}}$ & $\begin{array}{c}0.43 * * * \\
(0.09)\end{array}$ & $\begin{array}{c}0.36^{* * * *} \\
(0.12)\end{array}$ & $\begin{array}{c}0.71 * * * \\
(0.15)\end{array}$ & $\begin{array}{c}0.01 \\
(0.09)\end{array}$ & $\begin{array}{c}1.33^{* * * *} \\
(0.11)\end{array}$ & $\begin{array}{c}0.36^{* * * *} \\
(0.10)\end{array}$ & $\begin{array}{c}-0.15^{* *} \\
(0.08)\end{array}$ \\
\hline $\operatorname{gdp}^{\mathrm{m}}$ & $\begin{array}{c}0.35^{* * *} * \\
(0.12)\end{array}$ & $\begin{array}{c}-0.21^{* *} \\
(0.10)\end{array}$ & $\begin{array}{c}-0.23^{*} \\
(0.13)\end{array}$ & $\begin{array}{l}0.14^{*} \\
(0.08)\end{array}$ & $\begin{array}{l}-0.31 \\
(0.19)\end{array}$ & $\begin{array}{c}0.99^{* * *} * \\
(0.23)\end{array}$ & $\begin{array}{c}-0.19^{*} \\
(0.10)\end{array}$ \\
\hline $\operatorname{pop}_{\mathrm{x}}$ & $\begin{array}{l}-0.14 \\
(0.10)\end{array}$ & $\begin{array}{c}0.43^{* * * *} \\
(0.15)\end{array}$ & $\begin{array}{l}0.32 * \\
(0.17)\end{array}$ & $\begin{array}{l}-0.11 \\
(0.08)\end{array}$ & $\begin{array}{c}0.23 \\
(0.30)\end{array}$ & $\begin{array}{c}-0.41^{* * * *} \\
(0.14)\end{array}$ & $\begin{array}{c}0.52 * * * \\
(0.13)\end{array}$ \\
\hline pop $_{m}$ & $\begin{array}{c}-0.46^{* * * *} \\
(0.10)\end{array}$ & $\begin{array}{l}-0.13 \\
(0.20)\end{array}$ & $\begin{array}{c}-0.23^{* *} \\
(0.09)\end{array}$ & $\begin{array}{l}-0.08 \\
(0.12)\end{array}$ & $\begin{array}{c}-0.61 * * * \\
(0.12)\end{array}$ & $\begin{array}{c}-0.27 * * \\
(0.11)\end{array}$ & $\begin{array}{c}0.17 * * \\
(0.07)\end{array}$ \\
\hline Distance & $\begin{array}{c}-1.68^{* * * *} \\
(0.03)\end{array}$ & $\begin{array}{c}-1.93^{* * *} * \\
(0.05)\end{array}$ & $\begin{array}{c}-1.28^{* * * *} \\
(0.02)\end{array}$ & $\begin{array}{c}-1.61^{* * *} \\
(0.03)\end{array}$ & $\begin{array}{c}-1.51^{* * * *} \\
(0.08)\end{array}$ & $\begin{array}{c}-1.06^{* * * *} \\
(0.04)\end{array}$ & $\begin{array}{c}-1.85 * * * \\
(0.03)\end{array}$ \\
\hline Political* & $\begin{array}{c}0.04 * * * \\
(0.01)\end{array}$ & $\begin{array}{c}0.07 * * * \\
(0.01)\end{array}$ & $\begin{array}{c}0.06^{* * *} * \\
(0.01)\end{array}$ & $\begin{array}{c}0.00 \\
(0.01)\end{array}$ & $\begin{array}{c}0.12 * * * \\
(0.02)\end{array}$ & $\begin{array}{c}0.06 * * * \\
(0.01)\end{array}$ & $\begin{array}{c}0.08^{* * * *} \\
(0.01)\end{array}$ \\
\hline Latitude $_{\mathrm{x}}$ & $\begin{array}{c}-0.57 * * * \\
(0.04)\end{array}$ & $\begin{array}{l}-0.03 \\
(0.02)\end{array}$ & $\begin{array}{c}-0.11 * * * \\
(0.02)\end{array}$ & $\begin{array}{c}0.09 * * * \\
(0.01)\end{array}$ & $\begin{array}{c}-0.05^{*} \\
(0.03)\end{array}$ & $\begin{array}{c}0.01 \\
(0.01)\end{array}$ & $\begin{array}{c}0.12 * * * \\
(0.03)\end{array}$ \\
\hline Longitude $_{\mathrm{x}}$ & $\begin{array}{c}-0.11^{* * *} \\
(0.01)\end{array}$ & $\begin{array}{c}-0.01^{* * *} \\
(0.00)\end{array}$ & $\begin{array}{c}0.00 \\
(0.01)\end{array}$ & $\begin{array}{c}-0.01^{* * *} \\
(0.00)\end{array}$ & $\begin{array}{c}0.03^{* * * *} \\
(0.00)\end{array}$ & $\begin{array}{c}-0.03^{* * * *} \\
(0.01)\end{array}$ & $\begin{array}{c}-0.02 * * * \\
(0.01)\end{array}$ \\
\hline Year & $\begin{array}{c}0.05^{* * *} * \\
(0.01)\end{array}$ & $\begin{array}{c}0.05^{* * * *} \\
(0.01)\end{array}$ & $\begin{array}{c}0.06^{* * *} * \\
(0.00)\end{array}$ & $\begin{array}{c}0.05^{* * * *} \\
(0.01)\end{array}$ & $\begin{array}{c}0.05^{* * * *} \\
(0.01)\end{array}$ & $\begin{array}{c}0.05^{* * *} \\
(0.01)\end{array}$ & $\begin{array}{c}0.04 * * * \\
(0.00)\end{array}$ \\
\hline$R_{2}$ & 0.60 & 0.78 & 0.57 & 0.42 & 0.88 & 0.35 & 0.60 \\
\hline$N$ & 213,330 & 205,353 & 316,140 & 284,745 & 132,633 & 199,250 & 172,764 \\
\hline
\end{tabular}

Note: Standard errors in parentheses.

${ }^{*} p<.10$.

${ }^{* *} p<.05$.

$\stackrel{* * * *}{p}<.01$.

where $v_{i j}^{k}$ is the value of imports from country $i$ to country $j . \alpha_{i}$ and $b_{j}$ are importer and exporter fixed effects, GDP and population $(P o p)$ are defined for importers and exporters, distance ${ }_{i j}$ is the logged distance between countries, time is a time trend variable, longitude is the longitude for the exporting country, latitude is the latitude for the exporting country, and political represents the political stability of the exporting country (except for sugar, which has political stability for the importing country as in the ML application). These variables come from the same source as the ML application, that is, Gurevich and Herman (2018).

The econometric results from using the variables suggested by ML indicate high $R$-square for most commodities (Appendix Table A1). Most of the variables in the econometric model are statistically significant, and at the $0.01 \%$ level, but similarities and differences are visible from the $R$-square (e.g., rice versus soybean). Distance and the year trend are statistically significant at the $0.01 \%$ level for every model. The coefficient on the distance variable is negative as expected since longer distance involves more costs, and thus, decreasing the amount of trade that occurs. The magnitude of the distance coefficient is largest for corn, indicating that commodity is most affected by distance; while the -1.06 coefficient on sugar is the smallest. The time trend variable is positive and is between 0.04 and 0.06 , indicating that trade in these commodities is increasing over time. GDP is positive and statistically significant for many of the commodity-exporting countries, but the coefficient on GDP importer is mixed. Similarly, the coefficients on population (both the exporter and importer) are mixed. The political stability coefficient is positive and statistically significant for most commodities, that is, countries that are more political stable are more likely to be exporters. The coefficients on latitude and longitude (exporters) are also mixed. The largest beef, milk, and soy (beef) exporters tend to be in lower latitudes (longitudes).

As noted earlier, specifying gravity models remains a major challenge. Attempts were made to introduce pair-wise fixed effects, for example, origin-time and destination time, as in Yotov et al. (2016) as well as Correia et al. (2019). With several thousand such effects, convergence and multicollinearity, added to the above specification challenge. Compounding these issues, is the limited ability of gravity models to identify relative importance of explanatory variables (in the presence of fixed effects) as noted in the World Economic Outlook, IMF (2019). A deeper comparison of prescriptive ML models with high-dimension fixed effects specifications of PPML should be considered in future work.

Cite this article: Gopinath M, Batarseh FA, Beckman J, Kulkarni A and Jeong S (2021) International agricultural trade forecasting using machine learning. Data \& Policy, 3: e1. doi:10.1017/dap.2020.22 